\title{
Perineural but not systemic low-dose dexamethasone prolongs the duration of interscalene block with ropivacaine: a prospective randomized trial
}

\author{
This article was published in the following Dove Press journal: \\ Local and Regional Anesthesia \\ 8 April 2014 \\ Number of times this article has been viewed
}

\section{Ryosuke Kawanishi' \\ Kaori Yamamoto ${ }^{2}$ \\ Yoko Tobetto ${ }^{2}$ \\ Kayo Nomura ${ }^{2}$ \\ Michihisa Kato ${ }^{2}$ \\ Ritsuko Go² \\ Yasuo M Tsutsumi ${ }^{3}$ \\ Katsuya Tanaka ${ }^{3}$ \\ Yoshitsugu Takeda ${ }^{4}$}

'Department of Anesthesiology, Tokushima University Hospital,

Tokushima, ${ }^{2}$ Division of

Anesthesiology, Tokushima Red Cross Hospital, Komatsushima, ${ }^{3}$ Department of Anesthesiology, Institute of Health Bioscience, University of Tokushima Graduate School, Tokushima, ${ }^{4}$ Division of Orthopedics, Tokushima Red Cross Hospital, Komatsushima, Japan
Correspondence: Katsuya Tanaka Department of Anesthesiology, Institute of Health Bioscience, University of Tokushima Graduate School, 3-18-15 Kuramoto, Tokushima 770-8503, Japan $\mathrm{Tel}+81886337181$

Fax $+8 \mid 886337182$

Email katsuya.tanaka@tokushima-u.ac.jp
Purpose: To determine the effects of intravenous and perineural dexamethasone on the duration of interscalene brachial plexus block (ISB) with ropivacaine in patients undergoing arthroscopic shoulder surgery.

Patients and methods: In this prospective, randomized, placebo-controlled trial, patients presenting for arthroscopic shoulder surgery with an ISB were randomized to receive ropivacaine $0.75 \%$ (group C), ropivacaine $0.75 \%$ plus perineural dexamethasone $4 \mathrm{mg}$ (group Dperi), or ropivacaine $0.75 \%$ plus intravenous dexamethasone $4 \mathrm{mg}$ (group Div). The primary outcome was the duration of analgesia, defined as the time between performance of the block and the first request for analgesic.

Results: Thirty-nine patients were randomized. The median times of sensory block in groups C, Dperi, and Div were 11.2 hours (interquartile range [IQR] 8.0-15.0 hours), 18.0 hours (IQR 14.5-19.0 hours), and 14.0 hours (IQR 12.7-15.1 hours), respectively. Significant differences were observed between groups Dperi and C $(P=0.001)$. Kaplan-Meier analysis for the first analgesic request showed significant differences between groups Dperi and $\mathrm{C}(P=0.005)$ and between groups Dperi and Div $(P=0.008)$, but not between groups $\mathrm{C}$ and Div.

Conclusion: Perineural but not intravenous administration of $4 \mathrm{mg}$ of dexamethasone significantly prolongs the duration of effective postoperative analgesia resulting from a single-shot ISB with ropivacaine $0.75 \%$.

Keywords: ropivacaine, interscalene nerve block, dexamethasone, postoperative pain, perineural

\section{Introduction}

Shoulder surgery, even if an arthroscopic procedure, can be very painful, and many patients cannot sleep during the night of surgery because of pain. Interscalene brachial plexus block (ISB) is widely accepted as the gold standard of analgesia in the management of acute pain after shoulder surgery. ${ }^{1}$ Although single-shot ISB is useful for pain relief during the early postoperative period, the duration of analgesia is not sufficient for a comfortable transition to oral analgesics. ${ }^{2}$ Continuous peripheral nerve blocks have been used to provide a period of extended analgesia. These techniques have generally failed to gain popularity, due to high rates of catheter migration, anesthetic leakage, and the utilization of health care personnel for follow-up.,4

Dexamethasone prolongs peripheral nerve block when added to short-acting local anesthetics. ${ }^{5}$ Intravenous dexamethasone has been shown to attenuate the postoperative 
need for analgesics in different clinical settings, including after orofacial, general, urological, and orthopedic surgery. ${ }^{6}$ Although intravenous and perineural administrations of $10 \mathrm{mg}$ of dexamethasone were shown to be equivalent in increasing the duration of analgesia provided by a singleshot ISB with ropivacaine, ${ }^{7}$ intraoperative injection of $8 \mathrm{mg}$ or $10 \mathrm{mg}$ of dexamethasone may lead to hyperglycemia. ${ }^{8,9}$ The adverse effects of dexamethasone may be minimized by administering a lower dose. For example, the duration of analgesia from single-shot ISB did not differ when $4 \mathrm{mg}$ and $8 \mathrm{mg}$ of dexamethasone were added to bupivacaine. ${ }^{10}$

We hypothesized that the combination of ISB and perineural administration of $4 \mathrm{mg}$ of dexamethasone would prolong the duration of analgesia longer than that of ISB and intravenous administration of dexamethasone $4 \mathrm{mg}$ or ISB alone. We therefore conducted a prospective, randomized, placebo-controlled study to evaluate the effects of intravenous or perineural dexamethasone $4 \mathrm{mg}$ on the duration of ISB with ropivacaine $0.75 \%$ for postoperative analgesia in patients undergoing arthroscopic shoulder surgery.

\section{Patients and methods}

The study protocol was reviewed and approved by the institutional review board at Tokushima Red Cross Hospital. All patients between ages 20 and 75 years undergoing arthroscopic shoulder surgery were eligible, and informed consent was obtained prior to inclusion. Patients who refused consent and those diagnosed with a clinical condition in which ISB was contraindicated were excluded, including patients with a history of coagulation disorder, an international normalized ratio $>1.5$, skin infection at the site of the block, preexisting neuropathy involving the upper limb, drug dependency, systemic steroid use within the previous 6 months, peptic ulcer disease, diabetes mellitus, renal disease, hepatic disease, or pregnancy.

All patients underwent ISB with $20 \mathrm{~mL}$ of ropivacaine $0.75 \%$. Using a closed-envelope method, patients were randomized to receive ropivacaine $0.75 \%$ (group C), ropivacaine $0.75 \%$ plus perineural dexamethasone $4 \mathrm{mg}$ (group Dperi), or ropivacaine $0.75 \%$ plus intravenous dexamethasone $4 \mathrm{mg}$ (group Div).

Anesthesia was induced and maintained by intravenous administration of propofol $1 \mathrm{mg} \cdot \mathrm{kg}^{-1}$ and remifentanil (continuous infusion, $0.1-0.3 \mu \mathrm{g} \cdot \mathrm{kg}^{-1} \cdot$ minute $^{-1}$ ), rocuronium $\left(0.7 \mathrm{mg} \cdot \mathrm{kg}^{-1}\right)$, and sevoflurane $(1.0-1.5$ minimum alveolar concentration). All patients were intubated and ventilated with an oxygen/air admixture. After induction of anesthesia, all patients were administered intravenous morphine $5 \mathrm{mg}$ to minimize minor postoperative pain, including sore throat and back pain. ISB was performed after the surgical procedure by attending anesthesiologists skilled in the interscalene approach. An ultrasound-guided technique was used, along with $50 \mathrm{~mm}$-long nerve-block needles (Hakko, Tokyo, Japan). The ultrasound-guided technique consisted of an inplane posterior approach at the level of the cricoid cartilage. The nerve roots/trunks were identified as hypoechoic structures between the anterior and middle scalene muscles. Local anesthetic was injected $(0.75 \%$ ropivacaine $20 \mathrm{~mL}$ for groups $\mathrm{C}$ and Div, and $0.75 \%$ ropivacaine $20 \mathrm{~mL}$ and perineural dexamethasone $4 \mathrm{mg}$ for group Dperi), with the needle position readjusted as necessary to ensure appropriate spread. At the time of ISB, patients in group Div were administered dexamethasone $4 \mathrm{mg}$ intravenously. The criteria for failed ISB was shoulder pain soon after extubation of the endotracheal tube.

After extubation, all patients were transferred to the recovery room for at least 1 hour. Recovery room discharge criteria were stable vital parameters, absence of nausea and vomiting, and no further request for analgesics. On patient request, flurbiprofen was administered intravenously in the recovery room, and loxoprofen by mouth was administered after discharge from the recovery room. In our hospital, we explained to all patients not to endure pain after surgery, and to request analgesics as soon as pain developed. Therefore, we did not use pain scores when we administered analgesics.

The morning after surgery, all patients were assessed for pain by the numeric rating scale (NRS), motor block, and overall satisfaction. The timing and dosage of analgesics and the quality of sleep ( $1=$ no sleep disturbance because of pain or $2=$ sleep disturbance because of pain) were recorded. Overall patient satisfaction was graded from 1 (strong dissatisfaction) to 5 (strongly satisfied, and would recommend to others). During telephone interviews 28 days after surgery, patients were questioned about the late occurrence of any adverse event (eg, interscalene site infection or redness) and about neuropathy.

\section{Statistical analysis}

The primary end point was the length of the sensory block, defined as the time between the performance of the sensory block and the first administration of analgesia. Secondary outcomes were NRS the morning after surgery, analgesic need, sleep disturbance, and overall satisfaction score, measuring patient comfort. Following the removal of any identifying patient details (eg, name, date of birth, and social 
security number), data were entered into SPSS 20 (IBM, Armonk, NY, USA). Nonparametric data were analyzed using the Kruskal-Wallis test and Fisher's exact test. The Kaplan-Meier method was used for time-to-event analysis, from the time of ISB to the time of the first administration of analgesia, with curves compared using the log-rank test. Statistical significance was defined as a $P$-value $<0.05$. Unadjusted $P$-values were shown.

\section{Results}

From January to November 2012, 39 relatively health patients (American Society of Anesthesiologists physical status 1 or 2) were entered in the study. Four patients were excluded, three for unsuccessful blocks (because they complained of shoulder pain immediately after extubation), and one due to conversion to open surgery. Therefore, a total of 34 patients were included in the analysis: 12 each in groups $C$ and Dperi, and ten in group Div; their baseline characteristics were well balanced (Table 1).

Perineural dexamethasone $4 \mathrm{mg}$ significantly prolonged the duration of analgesia. The median duration of anesthesia was longer in group Dperi (18.0 hours, interquartile range [IQR] 14.5-19.0 hours) than in group C (11.2 hours, IQR 8.0-15.0 hours). The median duration of anesthesia was 14.0 hours (IQR 12.7-15.1 hours) in group Div. Significant differences were observed between group Dperi and $\mathrm{C}$ $(P=0.001)$ (Figure 1). Kaplan-Meier curves for the first analgesic request with patients not receiving any analgesics after 20 hours showed significant differences between groups Dperi and $\mathrm{C}(P=0.005)$, and between groups Dperi and Div $(P=0.008)$, but not between groups $\mathrm{C}$ and Div $(P=0.411)$ (Figure 2).

Although analgesic use did not differ among the three groups, there was a statistically significant between-group difference in NRS the morning after surgery $(P=0.03)$ (Figure 3). During the first night, $42 \%$ of patients in group $\mathrm{C}$ experienced sleep disturbance because of pain, compared

Table I Patient characteristics

\begin{tabular}{llll}
\hline Mean age (years) & $56.7 \pm 16.6$ & $55.6 \pm 12.8$ & $59.2 \pm 15.3$ \\
Sex, male/female $(\mathrm{n})$ & $8 / 4$ & $9 / 3$ & $7 / 3$ \\
Height $(\mathrm{cm})$ & $162.1 \pm 8.0$ & $162.2 \pm 10.3$ & $159.8 \pm 9.4$ \\
Weight $(\mathrm{kg})$ & $60.9 \pm 10.3$ & $66.3 \pm 9.9$ & $62.8 \pm 12.2$ \\
Duration of surgery (hours) & $172.9 \pm 54.4$ & $172.3 \pm 26.8$ & $178.0 \pm 47.1$ \\
Failed block $(\mathrm{n})$ & $\mathrm{I}$ & $\mathrm{I}$ & 2 \\
Converted to open & 0 & 0 & $\mathrm{I}$ \\
surgery $(\mathrm{n})$ & & & \\
\hline
\end{tabular}

Notes: Data are presented as means \pm standard deviation. There were no statistically significant differences among these variables.

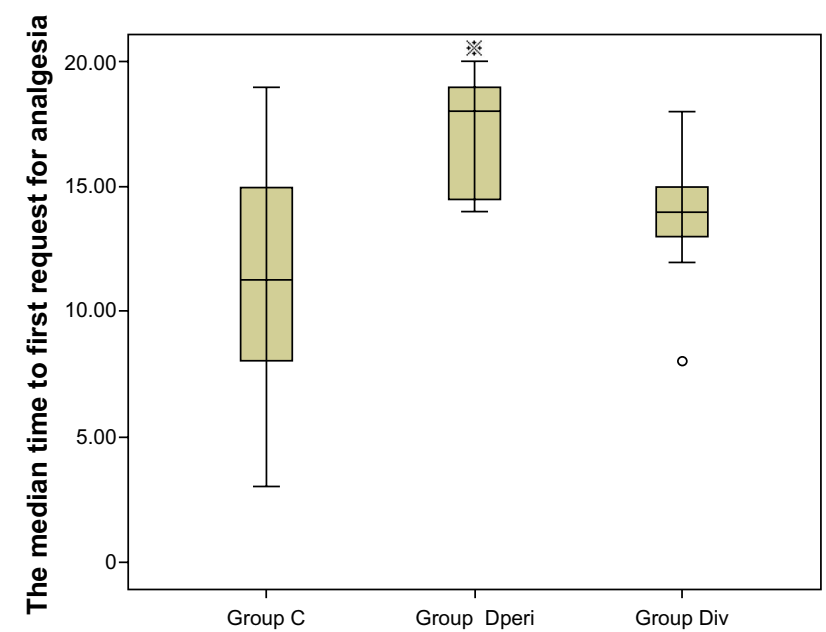

Figure I Median time to first request for analgesia (hours). Note: ${ }^{*} P=0.00$ I versus Group $C$. ${ }^{\circ}$ outlier.

with 0 in group Dperi (Table 2). Overall satisfaction scores of 4-5 were reported by $49 \%$ of patients, compared with $39 \%$ who responded neutrally and $14 \%$ with satisfaction scores of 1-2.

Patients were contacted 4 weeks after surgery. One patient in group Div complained of redness at the injection site. This redness disappeared gradually, and the patient required no further therapy.

\section{Discussion}

The present study demonstrates that a low dose $(4 \mathrm{mg})$ of perineural dexamethasone effectively prolongs the duration of ropivacaine ISB analgesia, with similar results observed when dexamethasone was administered intravenously or not administered at all. The duration of analgesia was increased to

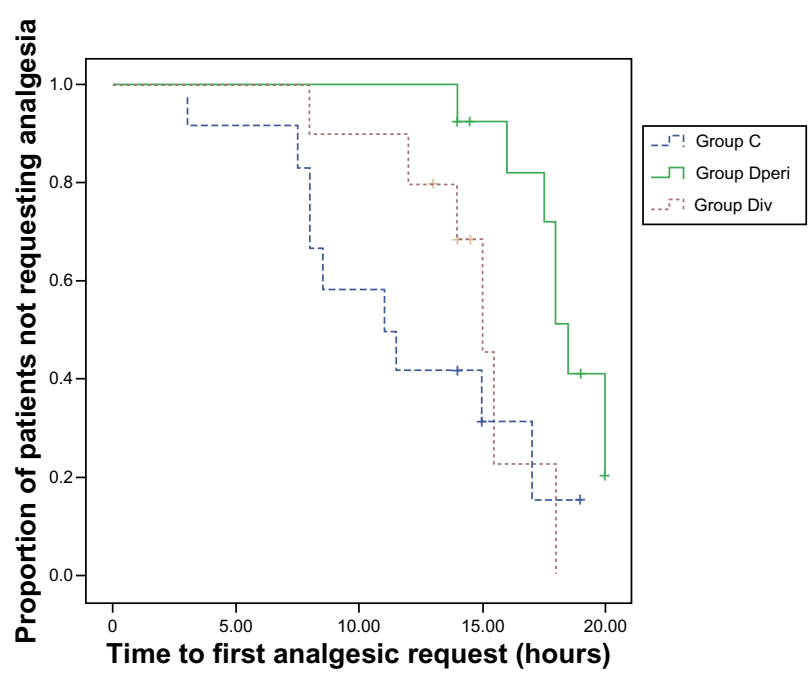

Figure 2 Kaplan-Meier analysis of time to first request for analgesia in the three patient groups. 


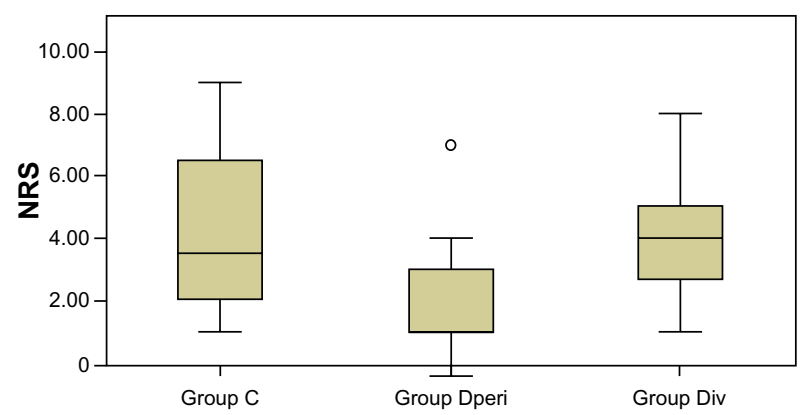

Figure 3 Numeric rating scale (NRS) the morning after surgery. Differences among the three groups were statistically significant, but differences between each pair of groups were not.

Note: ${ }^{\circ}$ outlier.

a median of 1.7-fold when dexamethasone was administered perineurally as an adjunct to ropivacaine $0.75 \%$, similar to previous results. ${ }^{7,10,11}$ Moreover, perineural dexamethasone reduced the incidence of sleep disturbances.

The precise mechanism by which dexamethasone prolongs the duration of sensory block is not completely understood. Although glucocorticoids have been reported to have a direct effect on nerves, other studies found that dexamethasone induced perineural vasoconstriction with concomitant slower absorption of local anesthetics. ${ }^{12,13}$ The rat sciatic nerve blockprolonging effect of dexamethasone could be eliminated by coadministration of the glucocorticoid-receptor antagonist cortexolone. ${ }^{14}$ The activity of dexamethasone appeared to be local, not systemic, because injections of dexamethasone at remote sites did not prolong blockade.

Dexamethasone has multiple systemic effects, such as reducing postoperative nausea, vomiting, and postoperative pain. Intravenous and perineural administration of $10 \mathrm{mg}$ of dexamethasone were shown to be equivalent in increasing the analgesic duration of ISB. ${ }^{7}$ De Oliveira et al reported in a meta-analysis that dexamethasone at doses higher than $0.1 \mathrm{mg} \cdot \mathrm{kg}^{-1}$ is an effective adjunct in multimodal strategies to reduce postoperative pain, while low-dose dexamethasone (less than $0.1 \mathrm{mg} \mathrm{kg}^{-1}$ ) does not decrease opioid consumption. ${ }^{6}$ We chose low-dose dexamethasone to minimize the possibility of such side effects of dexamethasone as hyperglycecemia. ${ }^{8,9}$ The dose of dexamethasone we used, $4 \mathrm{mg}$, was insufficient to have any systemic effect in reducing postoperative pain. We assume that this is why our result was different from Desmet et al. ${ }^{7}$

This study had several limitations. First, we did not assess the duration of sensory block in our patients using repeated neurological examinations, due to difficulties assessing sensory block after surgery. We therefore used the time until first analgesic request as a marker of the sensory block. Moreover, we were unable to determine if prolonged analgesia was associated with a prolongation of the sensory block. For patients, pain is the most important parameter defining satisfaction after surgery.

Second, we observed that the success rate of our block was 91.6\%, lower than reported previously. ${ }^{15}$ However, despite ISB being successful, 19\% of patients required opioids or reported pain while in the Postanesthesia Care Unit. We defined ISB as failed if a patient reported pain soon after extubation. However, most patients assessed as having failed block were unable to move their fingers and reported numbness in their thumbs. Although we used $20 \mathrm{~mL}$ ropivacaine, this may be insufficient. Other studies have used $\geq 30 \mathrm{~mL}$ local anesthetic to ensure ISB. ${ }^{7,10,11}$ Therefore, future studies should use larger amounts of ropivacaine to ensure ISB.

Thirdly, there are safety concerns regarding the perineural use of dexamethasone. However, corticosteroids have a long history of safe use in the epidural space for the treatment of radicular pain arising from nerve-root irritation. ${ }^{16}$ Therefore, any neurological risk of dexamethasone appears to be small.

Finally, the sample size in the present study was not enough. In order to achieve a power of $80 \%$ for three pair-wise comparisons, we required 15 patients per group; however, we had fewer per group in the present study. Therefore, the power of the present study is less than $80 \%$.

In conclusion, this study demonstrates that perineural but not intravenous administration of dexamethasone at a dose of $4 \mathrm{mg}$ significantly prolongs the duration of effective

Table 2 Secondary outcome parameters

\begin{tabular}{|c|c|c|c|c|}
\hline & Group C $(n=12)$ & Group Dperi $(n=\mid 2)$ & Group Div $(n=10)$ & $P$-value \\
\hline Mean loxoprofen use $(\mathrm{g})$ at 20 hours & 27 & 12.5 & 17.5 & NS \\
\hline Sleep disturbance at 20 hours ( $n$ ) & $5 / 7$ & $0 / 12$ & $2 / 10$ & 0.021 \\
\hline Mean NRS the morning after surgery & 4.4 & 2.3 & 4.1 & 0.03 \\
\hline $\begin{array}{l}\text { Number of patients who experienced } \\
\text { nausea and vomiting }(n)\end{array}$ & 2 & 0 & I & NS \\
\hline Mean satisfaction score $(I-5)$ at 20 hours & 3.3 & 3.3 & 4 & NS \\
\hline Incidence of dyspnea (n) & 0 & 0 & I & NS \\
\hline
\end{tabular}

Abbreviations: NRS, numeric rating scale; NS, not significant. 
postoperative analgesia resulting from a single-shot ISB with ropivacaine $0.75 \%$.

\section{Disclosure}

The authors report no conflicts of interest in this work.

\section{References}

1. Fredrickson MJ, Krishnan S, Chen CY. Postoperative analgesia for shoulder surgery: a critical appraisal and review of current techniques. Anaesthesia. 2010;65:608-624.

2. Brown DL, Ransom DM, Hall JA, Leicht CH, Schroeder DR, Offord KP. Regional anesthesia and local anesthetic-induced systemic toxicity: seizure frequency and accompanying cardiovascular changes. Anesth Analg. 1995;8:321-328.

3. Grossi P, Allegri M. Continuous peripheral nerve blocks: state of the art. Curr Opin Anesthesiol. 2005;18:522-526.

4. Litz RJ, Vicent O, Wiessner D, Heller AR. Misplacement of a psoas compartment catheter in the subarachnoid space. Reg Anesth Pain Med. 2004;29:60-64.

5. Hong JY, Han SW, Kim WO, Kim EJ, Kil HK. Effect of dexamethasone in combination with caudal analgesia on postoperative pain control in day-case paediatric orchiopexy. Br J Anaesth. 2010;105:506-510.

6. De Oliveira GS, Almeida MD, Benzon HT, McCarthy RJ. Perioperative single dose systemic dexamethasone for postoperative pain: a meta-analysis of randomized controlled trials. Anesthesiology. 2011;115:575-588.

7. Desmet M, Braems H, Reynvoet M, et al. IV and perineural dexamethasone are equivalent in increasing the analgesic duration of a single-shot interscalene block with ropivacaine for shoulder surgery: a prospective, randomized, placebo-controlled study. $\mathrm{Br} J$ Anaesth. 2013;111:445-452.
8. Eberhart LH, Graf J, Morin AM, et al. Randomised controlled trial of the effect of oral premedication with dexamethasone on hyperglycaemic response to abdominal hysterectomy. Eur J Anesthesiol. 2011;28: 195-201.

9. Pasternak JJ, McGregor DG, Lanier WL. Effect of single-dose dexamethasone on blood glucose concentration in patients undergoing craniotomy. J Neurosurg Anesthesiol. 2004;16:122-125.

10. Merle NT, Liang F, Sergei K, Alexander K, Nader DN. Adjuvant dexamethasone with bupivacaine prolongs the duration of interscalene block: a prospective randomized trial. J Anesth. 2011;25:704-709.

11. Cummings KC 3rd, Napierkowski DE, Parra-Sanchez I, et al. Effect of dexamethasone on the duration of interscalene nerve blocks with ropivacaine or bupivacaine. Br J Anaesth. 2011;107:446-453.

12. Wang PH, Tsai CL, Lee JS, Wu KC, Cheng KI, Jou IM. Effects of topical corticosteroids on the sciatic nerve: an experimental study to adduce the safety in treating carpal tunnel syndrome. J Hand Surg Eur Vol. 2011;36:236-243.

13. Shishido H, Kikuchi S, Heckman H, Myers RR. Dexamethasone decreases blood flow in normal nerves and dorsal root ganglia. Spine. 2002;27:581-586.

14. Castillo J, Curley J, Hotz J, et al. Glucocorticoids prolong rat sciatic nerve blockade in vivo from bupivacaine microspheres. Anesthesiology. 1996;85:1157-1166.

15. Davis JJ, Swenson JD, Greis PE, Burks RT, Tashjian RZ. Interscalene block for postoperative analgesia using only ultrasound guidance: the outcome in 200 patients. J Clin Anesth. 2009;21:272-277.

16. Price C, Arden N, Coglan L, Rogers P. Cost-effectiveness and safety of epidural steroids in the management of sciatica. Health Technol Assess. 2005;9:1-58.
Local and Regional Anesthesia

\section{Publish your work in this journal}

Local and Regional Anesthesia is an international, peer-reviewed, open access journal publishing on the development, pharmacology, delivery and targeting and clinical use of local and regional anesthetics and analgesics. The journal welcomes submitted papers covering original research, basic science, clinical studies, reviews \& evaluations,

\section{Dovepress}

guidelines, expert opinion and commentary, case reports and extended reports. The manuscript management system is completely online and includes a very quick and fair peer-review system, which is all easy to use. Visit http://www.dovepress.com/testimonials.php to read real quotes from published authors. 